\title{
Dynamic Changes of Antibodies to SARS-CoV-2 in Close Contacts
}

\author{
Jinjian Yao',* \\ Hangfei Wang ${ }^{2} * *$ \\ Weiling $\mathrm{Yu}^{3, *}$ \\ Yimin $\mathrm{Li}^{4}$ \\ Zhiqian Luo ${ }^{2}$ \\ Biao $\mathrm{Wu}^{5}$ \\ Wei Zhang ${ }^{6}$ \\ Xiaoran Liu $^{2}$ \\ Chuanzhu Lv 6,7
}

'Emergency Center, Hainan General Hospital Affiliated to Hainan Medical University, Haikou, Hainan, People's Republic of China; ${ }^{2}$ Department of Emergency, The First Affiliated Hospital of Hainan Medical University, Haikou, Hainan, People's Republic of China; ${ }^{3}$ Oncology Department, Haikou City People's Hospital, Haikou, Hainan, People's Republic of China; ${ }^{4}$ Department of General Surgery, Yang Jiang Hospital of Qiongzhong, Qiongzhong, Hainan, People's Republic of China; ${ }^{5}$ Department of Infectious Disease, Hainan General Hospital Affiliated to Hainan Medical University, Haikou, Hainan, People's Republic of China; ${ }^{6}$ Emergency and Trauma College of Hainan Medical University, Haikou, Hainan, People's Republic of China; ${ }^{7}$ Key Laboratory of Emergency and Trauma, Ministry of Education of Hainan Medical University, Haikou, Hainan, People's Republic of China

*These authors contributed equally to this work

Correspondence: Xiaoran Liu Department of Emergency, The First Affiliated Hospital of Hainan Medical University, Haikou, Hainan, People's Republic of China

Tel +86I3I I890I829

Email liuxiaoran3192@I63.com

Chuanzhu Lv

Key Laboratory of Emergency and Trauma,

Ministry of Education of Hainan Medical

University, Haikou, Hainan, People's Republic of China

Tel +86 I778983077I

Email Ivchuanzhu677@I26.com
Introduction: Close contacts of individuals with COVID-19 may directly gain immunity against SARS-CoV-2 despite lacking a detectable infection. This study examined SARS-CoV -2-specific antibodies levels based on gender, age, and exposure source in close contacts of individuals with COVID-19 and compared antibody levels to patients with an asymptomatic or symptomatic COVID-19 infection.

Methods: Two patients had confirmed COVID-19 infections at a community hospital in Qiongzhong, Hainan province. Contact tracing identified all individuals in the community who had been exposed to the two patients during the 14 days before their diagnoses. Close contacts quarantined for 14 days, underwent two SARS-CoV-2 tests, and were screened for SARS-CoV-2-specific antibodies at 7 and 12 weeks after the end of quarantine. SARS-CoV -2-specific antibody levels for the close contacts were compared to those for patients with an asymptomatic or symptomatic COVID-19 infection at 7 and 12 weeks after their diagnoses. Results: Contact tracing identified 10,573 individuals in the community, including 360 $(3.4 \%)$ close contacts. At 7 weeks, $30(8.33 \%)$ close contacts were positive for SARSCoV-2-specific antibodies ( $\operatorname{IgG}, \mathrm{n}=26$ [7.22\%]; IgM, $\mathrm{n}=4$ [1.11\%]), which were lower than the proportion of patients with an asymptomatic (IgG, 100\% [12/12]) or symptomatic (IgG, 93.6\% [44/47]) COVID-19 infection. SARS-CoV-2-specific IgM antibody levels were significantly higher in close contacts who were exposed through a relative compared to a doctor-patient relationship $(P=0.032)$. SARS-CoV-2-specific IgG antibody levels were significantly higher in close contacts aged $<18$ years vs $18-64$ years $(P=0.014)$. At 12 weeks, SARS-CoV-2-specific IgG antibody levels among close contacts were significantly lower than among patients with an asymptomatic $(P=0.004)$ or symptomatic COVID-19 infection $(P<0.001)$.

Conclusion: Immune protection conferred by close contact is short term and unlikely to contribute to herd immunity. There remains an unmet public health need for mass vaccination of populations to increase levels of protective antibodies and achieve and maintain herd immunity.

Keywords: SARS-CoV-2, COVID-19, antibody, close contact

\section{Introduction}

Severe acute respiratory syndrome coronavirus 2 (SARS-CoV-2) has spread across the globe at an unprecedented speed. ${ }^{1}$ Worldwide, there have been more than 1.4 billion confirmed cases of COVID-19, and over 3 million individuals have died. ${ }^{1,2}$ The epidemic persists due to lack of compliance with public health measures and inequitable vaccination distribution. The number of infections and deaths is growing exponentially in some regions, with regional resurgences elsewhere due to importation of the virus by travelers. In particular, individuals with clinically 
mild symptoms or asymptomatic infections are unlikely to be tested, but are able to transmit the disease through close contact. ${ }^{3}$ Serology screening demonstrates that seroprevalence estimates of SARS-CoV-2 are greater than the number of reported cumulative COVID-19 cases. ${ }^{4}$ Previous research has investigated the diagnostic utility of SARSCoV-2 -specific antibody detection in individuals with asymptomatic and symptomatic COVID-19 infections. ${ }^{5-7}$ However, the characteristics and kinetics of antibodies against SARS-CoV-2 in close contacts of individuals with COVID-19, or whether SARS-CoV-2 seropositivity after close contact will prevent infection on re-exposure and contribute to herd immunity, is not known.

Evidence suggests that close contacts of individuals with COVID-19 are able to gain immunity against SARSCoV-2 despite lacking a detectable infection. ${ }^{8}$ The objective of this study was to examine the characteristics and kinetics of SARS-CoV-2-specific antibodies based on gender, age, and exposure source in close contacts of individuals with COVID-19, and compare antibody titers and duration of immunity to patients with asymptomatic and symptomatic COVID-19 infections. Findings will characterize the immune response after close contact and inform the strategic design of COVID-19 vaccination programs.

\section{Methods}

\section{Study Design and Close Contract Tracing}

Data for this study were collected from a hospital that provides healthcare to the community in Qiongzhong, at the center of Hainan province, China. The community comprises 10,573 individuals across $105.99 \mathrm{~km}^{2}$. Between January 18 and January 20, 2020, two patients at the hospital had confirmed COVID-19 infections. Epidemiological investigations revealed one patient was diagnosed with severe COVID-19 following close contact with an individual with COVID-19, and one patient was diagnosed with mild COVID-19 following contact with an individual who had traveled from Wuhan. The timeline for this study is provided in Supplementary Figure 1.

Contact tracing was used to identify all individuals who may have been exposed to these case-patients during the 14 days before their diagnoses were confirmed. Close contacts were defined as individuals who had an unprotected face-to-face interaction with an infected patient, including: 1) being within 2 meters of the case-patient for more than 10 minutes; 2) sharing a living area or healthcare waiting room with the case-patient or for $\geq 2$ hours after the case-patient was present; and/or 3) having contact with the case-patient's secretions (eg, being coughed on when unprotected, touching used paper tissues with a bare hand). Demographic and epidemiological data for close contacts, including contact and exposure history and symptoms, were recorded. Close contacts were quarantined for 14 days, during which they were monitored for COVID-19 related signs and symptoms and underwent two viral nucleic acid tests on Day 2 and Day 13. Close contacts were screened for SARS-CoV-2-specific antibodies and underwent additional viral nucleic acid tests at 7 and 12 weeks after the end of their quarantine. SARS-CoV -2-specific antibody levels for the close contacts were compared with those for patients with an asymptomatic or symptomatic COVID-19 infection at 7 and 12 weeks after they were diagnosed with a viral nucleic acid test. Asymptomatic patients had epidemiological evidence of COVID-19, were positive for SARS-CoV-2 RNA on RTPCR, and had no COVID-19 related clinical symptoms and CT imaging findings indicative of viral pneumonia. Symptomatic patients had epidemiological evidence of COVID-19, were positive for SARS-CoV-2 RNA on RTPCR, and had fever, respiratory symptoms or other COVID-19 related clinical symptoms, CT imaging findings indicative of viral pneumonia and/or decreased lymphocyte counts.

The study was approved by the Hainan General Hospital Ethics Committee in accordance with the Declaration of Helsinki (Ethical approval No. Med-EthRe [2021] 190). Written informed consent was obtained from all study participants.

\section{Serum SARS-CoV-2 Specific lgG/lgM Antibodies Assay}

A commercial magnetic chemiluminescence enzyme immunoassay (MCLIA) (Bioscience. Co., Ltd., Tianjin; No: 20203400183 [IgG], 20203400182 [IgM]; approved by the China National Medical Products Administration) was used to measure SARS-CoV-2-specific IgG and IgM levels in venous blood obtained from close contacts and patients with an asymptomatic or symptomatic COVID-19 infection, according to the manufacturer's instructions. The MCLIA for IgG or IgM detection was developed based on a double-antibody sandwich immunoassay. Recombinant antigens containing the nucleoprotein and a peptide from the spike protein of SARS-CoV-2 were conjugated with fluorescein isothiocyanate (FITC) and 
immobilized on anti-FITC antibody-magnetic beads, as previously described. $50 \mu \mathrm{L}$ activated magnetic beads coated with SARS-CoV-2 antigens were incubated for 15 min at $37^{\circ} \mathrm{C}$. Chemiluminescence was detected (Axceed 260 , Bioscience) after beads were incubated with acridinium ester-labeled mouse anti-human IgM or IgG antibody and hydrogen peroxide. Assays were calibrated using various dilutions of inactivated serum from each study participant to establish a standard linear correlation between light intensity and relative antibody level. Relative antibody levels were calculated as measured chemiluminescence divided by the constant derived from the linear correlation. Antibody levels were expressed as the ratio of the signal to the cutoff $(\mathrm{S} / \mathrm{CO})$ value, where an $\mathrm{S} /$ $\mathrm{CO}>1$ was considered positive and $\mathrm{S} / \mathrm{CO}<1$ was considered negative. Strict biosafety and quality control protocols were used to ensure stable and uniform experimental conditions and robust data.

\section{Viral SARS-CoV-2 Detection}

Reverse transcription-polymerase chain reaction (RT-PCR) was used to detect SARS-CoV-2 nucleic acid (SARS-CoV -2 open reading framelab [ORF1ab] and nucleocapsid [N] genes) in nasopharyngeal swabs obtained from close contacts and patients with an asymptomatic or symptomatic Covid-19 infection. Total nucleic acids were extracted from the nasopharyngeal swabs of the study participants. Target genes (ORF1ab and N) were simultaneously amplified and tested. Primers were developed according to the recommendations of the Chinese CDC (ORF1ab forward: CCCTGTGGGTTTTACACTTAA, ORF1ab reverse: ACGATTGTGCATCAGCT GA, ORF1ab probe: 5'CCGTCTGCGGTATGTGGAAAGGTTATGG-3' (FAM dye labeled); $\mathrm{N}$ forward: GGGGAACTTCTCC TGCTAGAAT, $\mathrm{N}$ reverse: CAGACATTTTGCTCTCA AGCTG, N probe: 5'-TTGCTGCTGCTTGACAGATT-3' (VIC dye labeled)). PCR conditions were: $50^{\circ} \mathrm{C}$ for 15 $\min , 95^{\circ} \mathrm{C}$ for $15 \mathrm{~min}, 45$ cycles of $94^{\circ} \mathrm{C}$ for $15 \mathrm{~s}, 55^{\circ} \mathrm{C}$ for $45 \mathrm{~s}$ (fluorescence collection). $\mathrm{Ct}$ values $<37$ or $>40$ were defined as positive and negative, respectively.

\section{Statistical Analysis}

Statistical analyses were performed using SPSS (v 3.6.2). Categorical variables are reported as frequencies or percentages and were compared with the Wilcoxon rank-sum test. For continuous variables, normally distributed data are presented as mean \pm standard deviation and were compared with the independent sample $t$ test. Non-normally distributed data are presented as median (inter-quartile range $[\mathrm{IQR}]$ ) and were compared with the Kruskal-Wallis $H$-test. $P<0.05$ was considered statistically significant.

\section{Results}

\section{Demographic and Epidemiological Characteristics of the Close Contacts}

Contact tracing identified 10,573 individuals, including 360 (3.4\%) close contacts, who had been exposed to the casepatients included in this study. Demographic and epidemiological characteristics of the close contacts are summarized in Table 1. Among the close contacts, there were 164 (45.5\%) males and $196(54.4 \%)$ females. A total of 89 (24.7\%) close contacts were aged $<18$ years, $177(49.2 \%)$ close contacts were aged between 18 and 64 years, and 19 (5.3\%) close contacts were aged $\geq 65$ years. Regarding location of exposure, $68(18.9 \%)$ close contacts were exposed in the hospital setting, and $292(81.1 \%)$ close contacts were exposed in the community (social) setting. A total of $279(77.5 \%)$ close contacts were exposed through a doctor-patient relationship, while 68 (18.8\%) close contacts were colleagues and 13 (3.6\%) close contacts were family members of the case patients.

During quarantine, 360 close contacts were negative for SARS-CoV-2 on nucleic-acid-based testing. At 7 weeks of follow-up, $30(8.33 \%)$ close contacts were positive for SARS-CoV-2-specific antibodies $(\mathrm{S} / \mathrm{CO} \geq 1)$, including 26 $(7.22 \%)$ close contacts who were positive for SARS-CoV -2-specific IgG antibody and $4(1.11 \%)$ close contacts who were positive for SARS-CoV-2-specific IgM antibody. At 12 weeks of follow-up, 3 close contacts were positive for SARS-CoV-2-specific IgG antibody.

\section{Overall Immunological Assessment}

At 7 weeks of follow-up, among the 360 close contacts, median (IQR) SARS-CoV-2-specific IgG antibody levels were 0.18 (0.14-0.29), and median (IQR) SARS-CoV-2-specific IgM antibody levels were $0.15(0.08-0.28)$. SARS-CoV-2-specific IgG (median S/CO, 0.33; IQR,0.16-0.60 vs 0.18; IQR,0.15$0.36 ; P=0.01$ ) and $\operatorname{IgM}$ (median $\mathrm{S} / \mathrm{CO}, 0.25 ; \mathrm{IQR}, 0.10-0.62$ vs median S/CO, 0.16; IQR,0.09-0.33; $P=0.02$ ) antibody levels were significantly higher in close contacts who were exposed in the hospital setting compared to the social setting (Figure 1A and B). SARS-CoV-2-specific IgG and IgM antibody levels were significantly higher in close contacts aged $<18$ years compared to close contacts aged 18-64 years $(P=0.014)$ (Figure 1C and D). There was no significant difference in SARS-CoV-2-specific IgG antibody levels in close contacts 
Table I Demographic and Epidemiological Characteristics of the Close Contacts $(n=360)$

\begin{tabular}{|c|c|c|c|}
\hline Characteristics & All Close Contacts & Seropositive & Seronegative \\
\hline Male \% (n) & $45.5 \%(164 / 360)$ & $3.6 \%(13 / 360)$ & $41.9 \%(151 / 360)$ \\
\hline Female \% (n) & $54.4 \%(196 / 360)$ & $4.7 \%(17 / 360)$ & $49.7 \%(179 / 360)$ \\
\hline Age, years mean $( \pm S D)$ & $31.5 \pm 23$ & $22.7 \pm 23.8$ & $32.38 \pm 22.8$ \\
\hline$<18 \%(n)$ & $32.8 \%(118 / 360)$ & $56.7 \%(17 / 30)$ & $27.0 \%(77 / 285)$ \\
\hline $18-64 \%(n)$ & $61.1 \%(220 / 360)$ & $36.7 \%(11 / 30)$ & $44.7 \%(161 / 360)$ \\
\hline$\geqq 65 \% \quad(n)$ & $6.1 \%(22 / 360)$ & $6.6 \%(2 / 30)$ & $6.0 \%(17 / 285)$ \\
\hline \multicolumn{4}{|l|}{ Setting } \\
\hline Hospital setting \% (n) & $18.6 \%(67 / 360)$ & $26.7 \%(8 / 30)$ & $16.4 \%(59 / 360)$ \\
\hline Social setting \% (n) & $81.1 \%(292 / 360)$ & $73 \%(22 / 30)$ & $75 \%(270 / 360)$ \\
\hline \multicolumn{4}{|l|}{ Source } \\
\hline Colleague \% (n) & $18.8 \%(68 / 360)$ & $30 \%(9 / 30)$ & $16.7 \%(60 / 360)$ \\
\hline Relative \% (n) & $3.6 \%(13 / 360)$ & $13.3 \%(4 / 30)$ & $2.5 \%(9 / 360)$ \\
\hline Physician-patient \% (n) & $77.5 \%(279 / 360)$ & $56.6 \%(17 / 30)$ & $74.2 \%(267 / 360)$ \\
\hline \multicolumn{4}{|l|}{ Antibody level } \\
\hline $\lg G(X \pm S)$ & $0.474 \pm 1.487$ & $2.780 \pm 4.580$ & $0.264 \pm 0.184$ \\
\hline $\lg M(X \pm S)$ & $0.320 \pm 0.498$ & $0.856 \pm 1.166$ & $0.271 \pm 0.349$ \\
\hline
\end{tabular}

who were exposed through a colleague, relatives or a doctorpatient relationship (Figure 2A). SARS-CoV-2-specific IgM antibody levels were significantly higher in close contacts who were exposed through a relative compared to a doctor-patient relationship $(P=0.032)$ (Figure $2 \mathrm{~B})$.

\section{Immunological Assessment in Seropositive Close Contacts (S/CO $\geq 1$ ) and Patients with COVID-I 9 at 7 Weeks of Follow-Up}

At 7 weeks of follow-up, $30(8.33 \%)$ close contacts were positive for SARS-CoV-2-specific antibodies, including 13 (43.3\%) males and 17 (56.6\%) females, with a median age of 10 years (range 1-78 years). Among these, 17 (56.7\%) close contacts were aged $<18$ years, $11(40.0 \%)$ close contacts were aged between 18 and 64 years, and 2 (6.7\%) close contacts were aged $\geq 65$ years (Table 1 ). Median S/CO (IQR) SARS-CoV-2-specific IgG antibody levels were 1.63 (1.112.19), and median S/CO (IQR) SARS-CoV-2-specific IgM antibody levels were $0.31(0.18-1.14)$ (Table 2). There were no significant differences in SARS-CoV-2-specific IgG and IgM antibody levels in close contacts who were exposed in the hospital setting compared to the community (social exposure) (Figure 3A and B).

SARS-CoV-2-specific antibody levels for the 30 close contacts were compared to those for 59 patients with
COVID-19, including 12 patients with an asymptomatic infection and 47 cases with a symptomatic infection.

At 7 weeks of follow-up, $100 \%$ of patients with an asymptomatic (12/12) COVID-19 infection and 93.6\% (44/ 47) of patients with a symptomatic COVID-19 infection were SARS-CoV-2-specific $\operatorname{IgG}$ positive $(\mathrm{S} / \mathrm{CO} \geq 1)$. Among patients with an asymptomatic COVID-19 infection, median S/CO (IQR) SARS-CoV-2-specific IgG antibody levels were 5.22 (2.260-16.318) and median S/CO (IQR) SARS-CoV-2-specific IgM antibody levels were 0.53 (0.243-1.668). Among patients with a symptomatic COVID-19 infection, median S/CO (IQR) SARS-CoV -2-specific IgG antibody levels were 24.4 (4.97-48.18), and median S/CO (IQR) SARS-CoV-2-specific IgM antibody levels were $0.41(0.16-1.10)$. SARS-CoV-2-specific IgG levels were significantly higher in patients with an asymptomatic COVID-19 infection or a symptomatic COVID-19 infection compared to close contacts $(P<0.001)$ (Figure 4A).

\section{Immunological Assessment in Seropositive Close Contacts and Patients with COVID-19 at 12 Weeks of Follow-Up}

At 12 weeks of follow-up, 90\% (27/30) of close contacts became seronegative $(\mathrm{S} / \mathrm{CO}<1)$, and only 3 close contacts remained seropositive $(P<0.001)$ (Figure $5 \mathrm{~A}$ and $\mathrm{B}$ ). SARS-CoV-2-specific IgG antibody levels were 
A

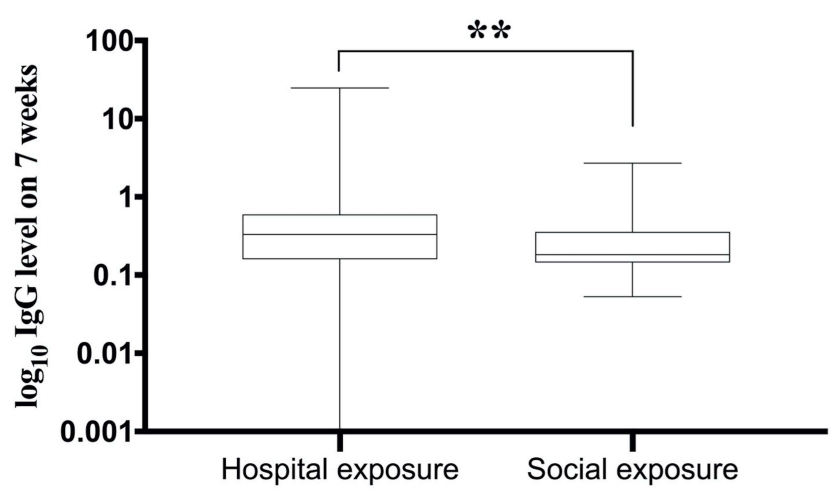

C

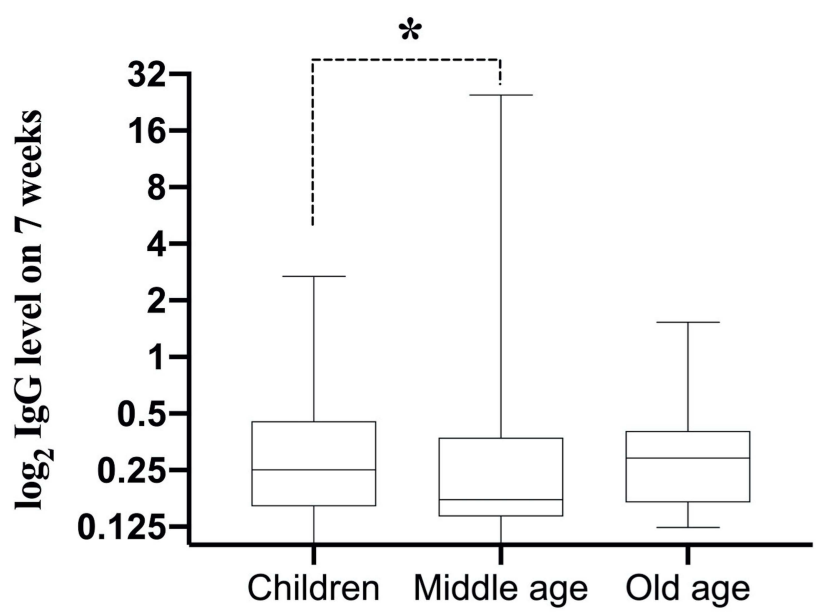

B

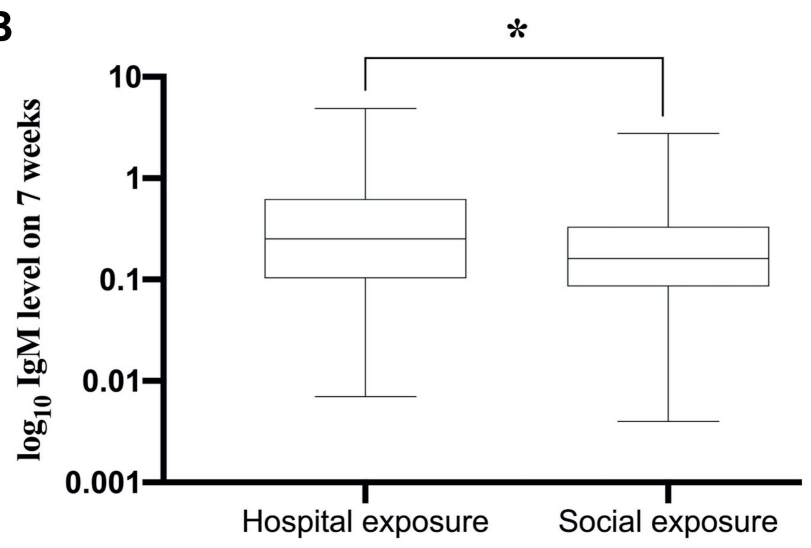

D

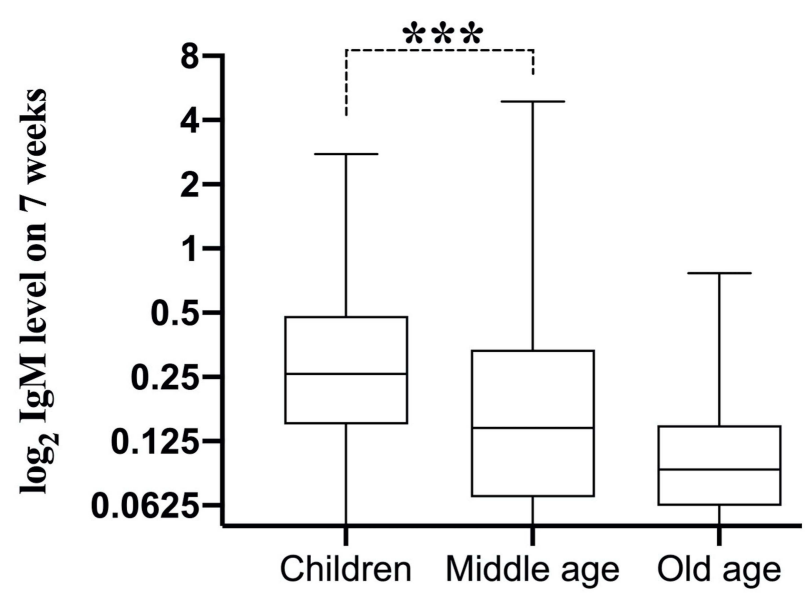

Figure I SARS-CoV-2-specific antibody levels in close contacts at 7 weeks of follow-up $(n=360)$. (A) SARS-CoV-2-specific lgG antibody levels in close contacts stratified by exposure setting (social exposure vs. hospital exposure, ${ }^{*} p=0.01$ ); (B) SARS-CoV-2-specific IgM antibody levels in close contacts stratified by exposure setting (social exposure vs. hospital exposure, ${ }^{*} p=0.02$ ); (C) SARS-CoV-2-specific IgG antibody levels in close contacts stratified by age (middle age vs children, ${ }^{*} p=0.014$ ); (D) SARS-CoV -2-specific IgM antibody levels in close contacts stratified by age (middle age vs children, ${ }^{* *} p=0.003$; old age vs children $* * * p<0.00 \mathrm{I}$ ).

significantly higher in close contacts who were exposed in the hospital setting compared to the community (social exposure) $(P=0.017)$ (Figure $3 \mathrm{C})$, while IgM antibody levels were close to significance $(P=0.05)$ (Figure 3D), SARS-CoV-2-specific IgG antibody levels were significantly higher in close contacts who were exposed through a colleague compared to a doctor-patient relationship $(P=0.042)$ (Figure 2C).

At 12 weeks of follow-up, among the 59 patients with COVID-19, SARS-CoV-2-specific antibody levels had decreased in $83.3 \%(10 / 12)$ of patients with an asymptomatic infection and $85.1 \%(40 / 47)$ of patients with a symptomatic infection. Two patients with an asymptomatic COVID-19 infection became seronegative, and 8.5\% (4/47) of patients with a symptomatic COVID-19 infection became seronegative. In patients with an asymptomatic
COVID-19 infection, there was no significant difference in SARS-CoV-2-specific IgG antibody levels at 12 weeks of follow-up compared to 7 weeks of follow-up $(P=0.59)$ (Figure 5C), while SARS-CoV-2-specific IgM antibody levels were significantly decreased $(P=0.003)$ (Figure 5D). In patients with a symptomatic COVID-19 infection, SARS-CoV-2-specific IgG antibody levels were significantly decreased at 12 weeks of follow-up compared to 7 weeks of follow-up $(P=0.029)$ (Figure 5E), but there was no significant difference in SARS-CoV-2-specific IgM antibody levels $(P=0.302)$ (Figure $5 \mathrm{~F}$ ). Median $\mathrm{S} / \mathrm{CO}$ (IQR) SARS-CoV-2-specific IgG antibody levels among close contacts $(0.56 ; 0.34-0.87)$ were significantly lower than among patients with an asymptomatic COVID-19 infection (3.69; 1.52-10.13) $(P=0.004)$ or a symptomatic COVID-19 infection (9.66; 3.34-21.23) $(P<0.001)$ (Figure 4B). 
A

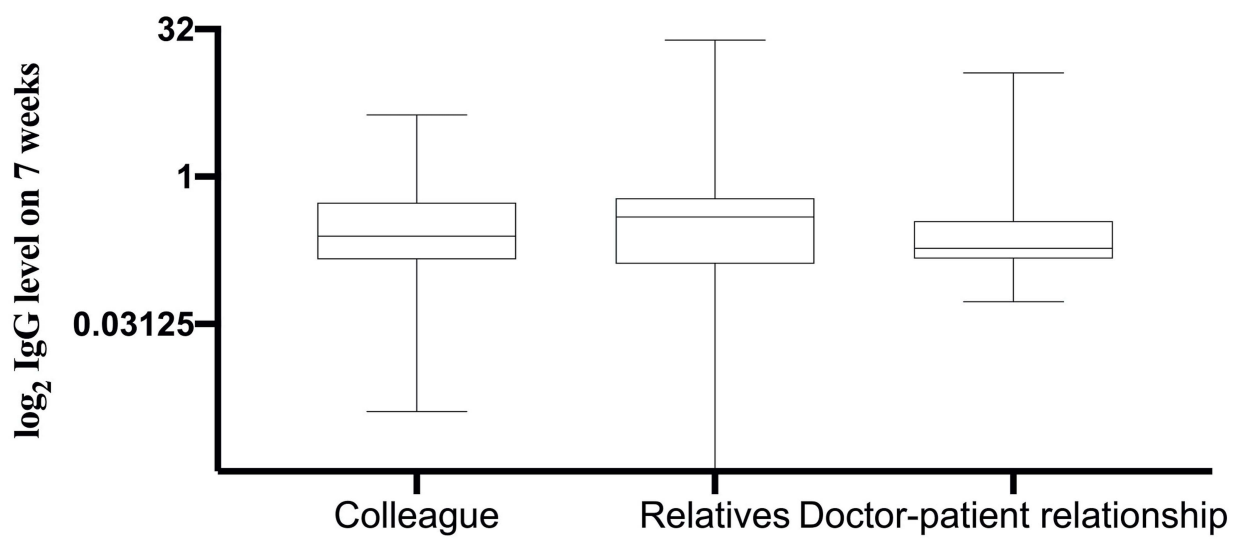

B

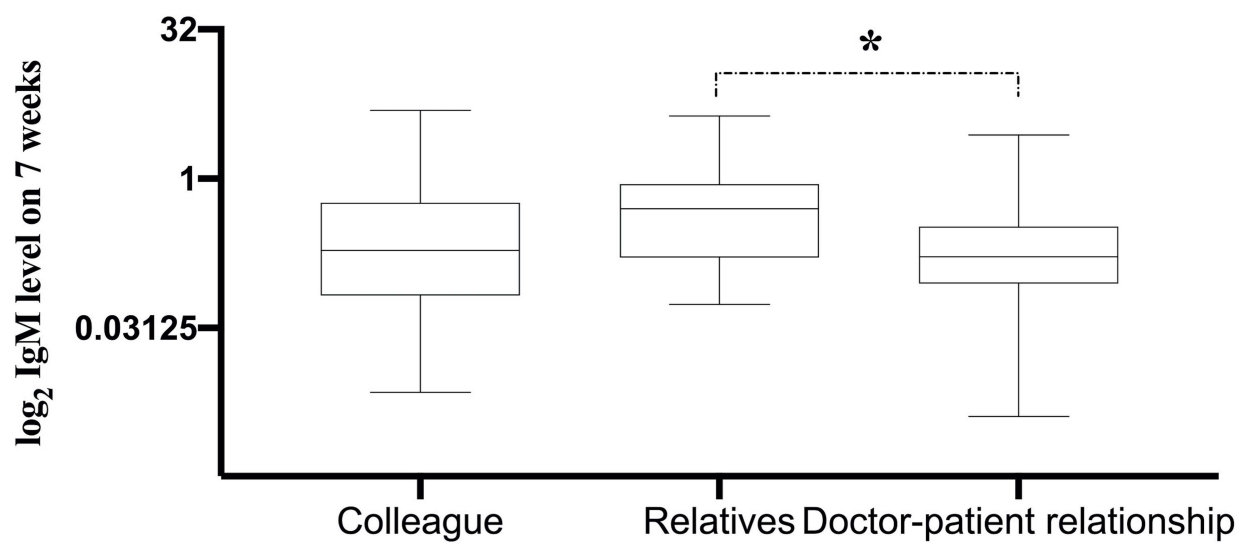

C

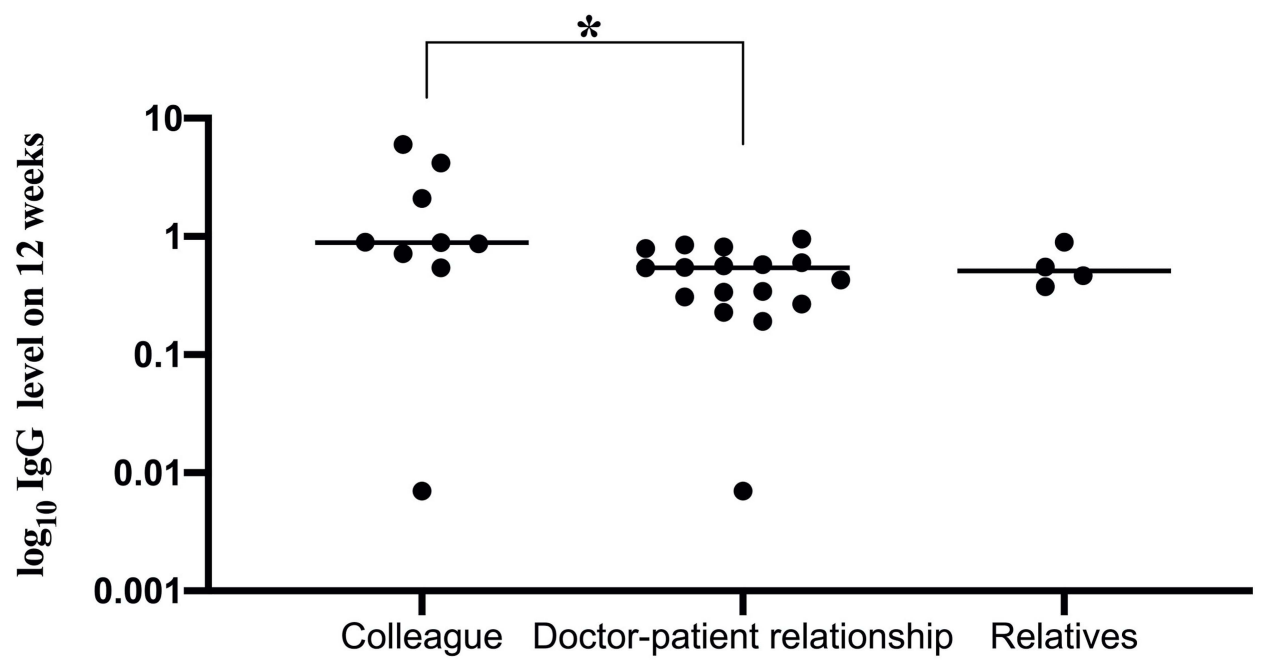

Figure 2 SARS-CoV-2-specific antibody levels in close contacts at 7 and 12 weeks of follow-up (A) SARS-CoV-2-specific lgG antibody levels in all close contacts at 7 weeks of follow-up stratified by source $(n=360)$; (B) SARS-CoV-2-specific IgM antibody levels in all close contacts at 7 weeks of follow-up stratified by source ( $n=360$ ) (relatives vs doctor-patient relationship, ${ }^{*} p=0.032$ ); (C) SARS-CoV-2-specific lgG antibody levels at I 2 weeks of follow up in close contacts who were seropositive (S/CO $\geq I$ ) at 7 weeks of follow-up, stratified by source $(n=30)$ (colleague vs doctor-patient relationship, ${ }^{*} p=0.042$ ). 
Table 2 Demographic and Epidemiological Characteristics of the Seropositive $(\mathrm{S} / \mathrm{CO} \geq \mathrm{I})$ Close Contacts at 7 Weeks of FollowUp $(n=30)$

\begin{tabular}{|l|l|}
\hline Characteristics & Close Contacts \\
\hline Male \% (n) & $43.3 \%(13 / 30)$ \\
\hline Female \% (n) & $56.7 \%(17 / 30)$ \\
\hline Age, years mean ( $\mathrm{SD})$ & \\
$<18 \%(\mathrm{n})$ & $53.2 \%(16 / 30)$ \\
$18-64 \%(\mathrm{n})$ & $36.7 \%(11 / 30)$ \\
$\geqq 65 \% \quad(\mathrm{n})$ & $6.6 \%(2 / 30)$ \\
\hline Setting & \\
Hospital setting \% (n) & $26.7 \%(8 / 30)$ \\
Social exposure \% (n) & $73 \%(22 / 30)$ \\
\hline Source & \\
Colleague \% (n) & $30 \%(9 / 30)$ \\
Relative \% (n) & $13.3 \%(4 / 30)$ \\
Physician-patient \% (n) & $56.6 \%(17 / 30)$ \\
\hline
\end{tabular}

\section{Discussion}

The global COVID-19 pandemic shows no sign of receding. SARS-CoV-2 is mainly spread by direct person-toperson transmission through close contact. Active

\section{A}

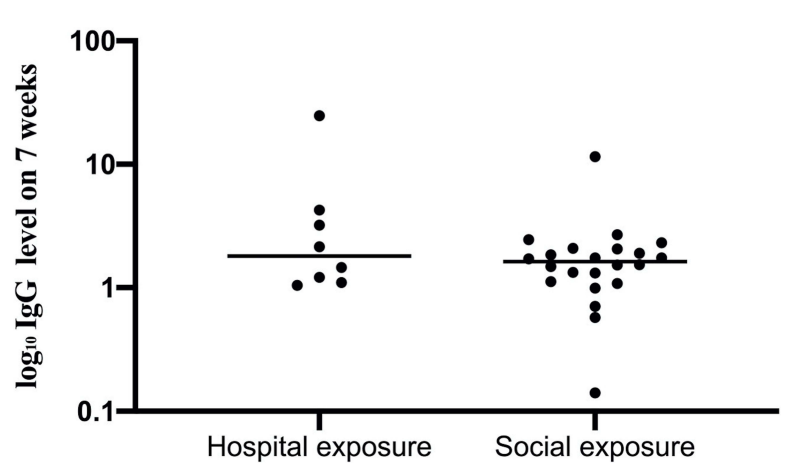

C

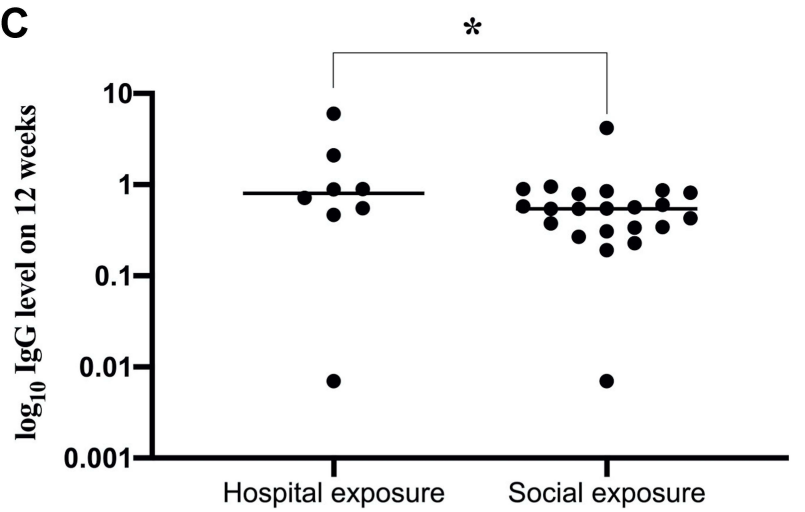

immunity can offer protection against COVID-19 through natural immunity after exposure to the disease, or through a vaccination. Knowledge of the antibody response to SARS-CoV-2 following close contact at the individual and population levels is limited.

The present study furthers our understanding of the mechanisms of immune control of SARS-CoV-2 and uncovers the dynamics of SARS-CoV-2 specific antibodies. Contact tracing was used to identify close contacts who had been exposed to two case-patients with confirmed COVID-19 infections. Close contacts were screened for SARS-CoV-2-specific antibodies at 7 and 12 weeks after the end of 14-day quarantine. SARS-CoV-2-specific antibody levels for the close contacts were compared with those for patients with an asymptomatic or symptomatic COVID-19 infection at 7 and 12 weeks after their diagnoses. These findings will inform strategies for the rational design and distribution of vaccines.

Results across our follow-up period showed that close contacts of individuals with COVID-19 developed a mild SARS-CoV-2-specific antibody response that was shortlived and decayed more rapidly than in patients with

B

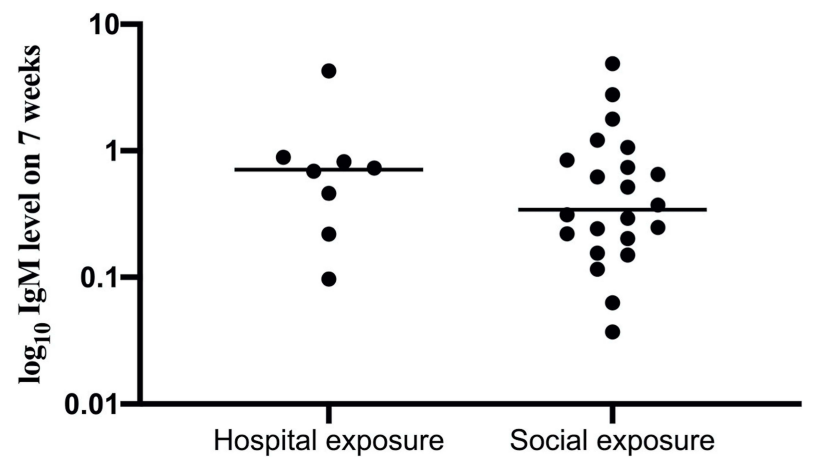

D

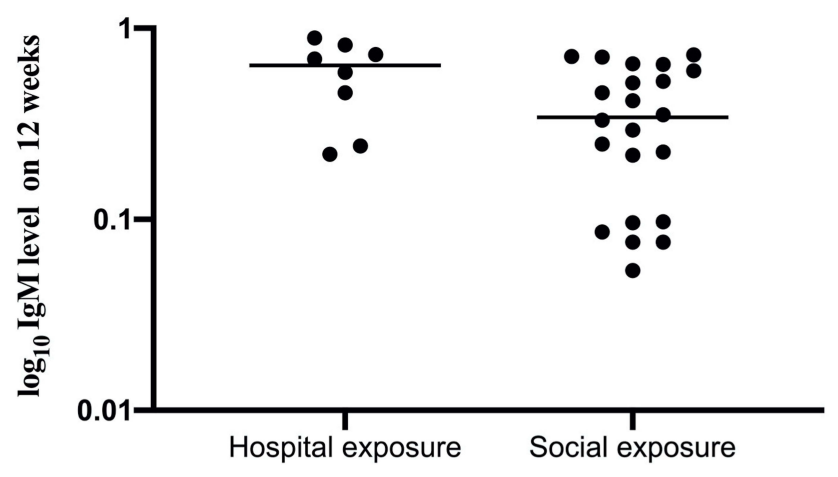

Figure 3 SARS-CoV-2-specific antibody levels at 7 and 12 weeks of follow-up in close contacts who were seropositive (S/CO $\geq 1$ ) at 7 weeks of follow-up. (A) SARS-CoV -2-specific lgG antibody levels at 7 weeks of follow-up stratified by exposure setting $(n=30)$; (B) SARS-CoV-2-specific lgM antibody levels at 7 weeks of follow-up stratified by exposure setting ( $n=30$ ); (C) SARS-CoV-2-specific lgG antibody levels at 12 weeks of follow-up stratified by exposure setting ( $n=30$ ) (hospital exposure vs social exposure, $\left.*_{p}=0.017\right)$; (D) SARS-CoV-2-specific lgM antibody levels at 12 weeks of follow-up stratified by exposure setting $(n=30)$. 

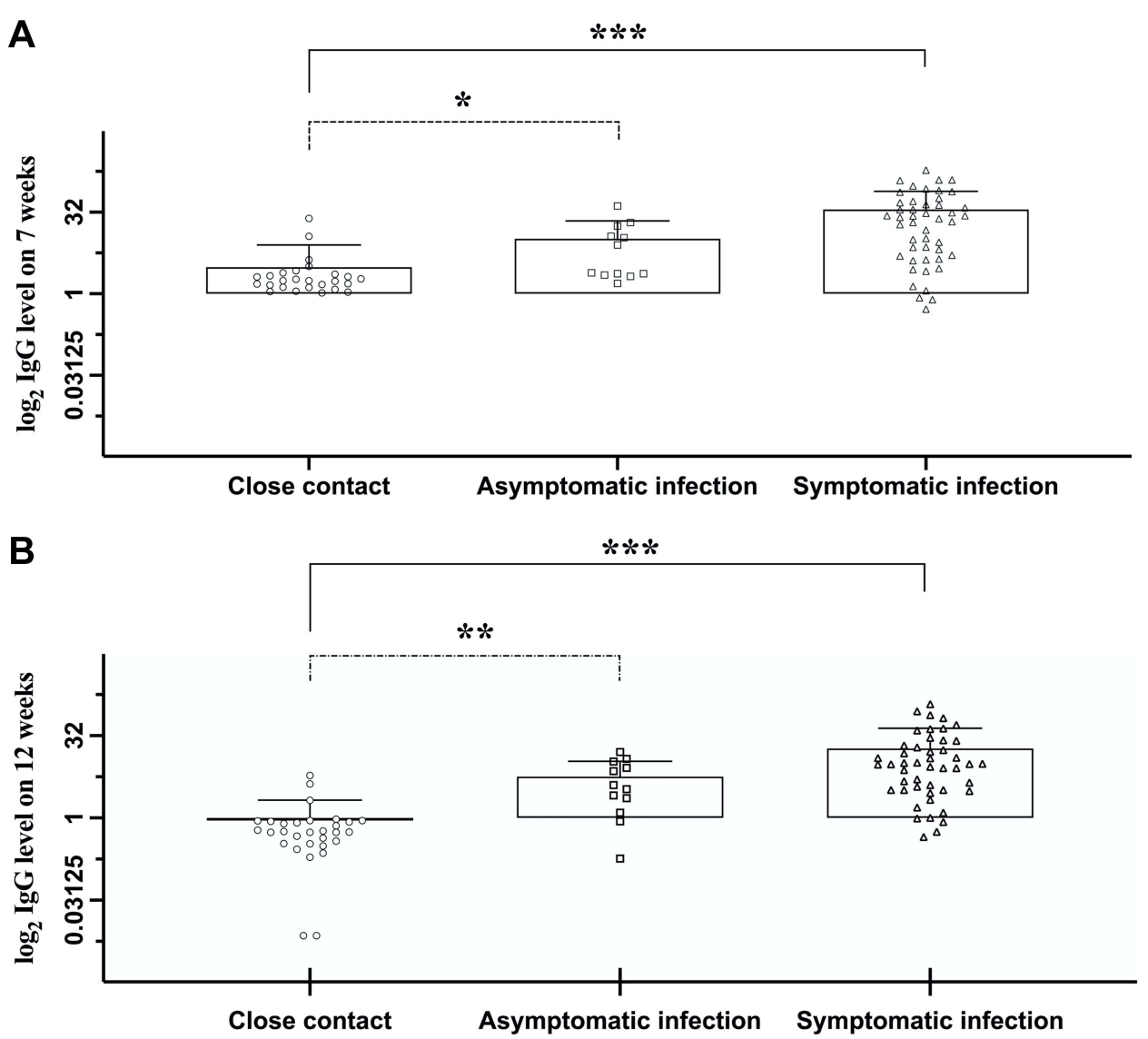

Figure 4 SARS-CoV-2-specific antibody levels at 7 and 12 weeks of follow-up in close contacts who were seropositive (S/CO $\geq 1)$ at 7 weeks of follow-up and patients with an asymptomatic or symptomatic COVID-19 infection. (A) SARS-CoV-2-specific lgG antibody levels in close contacts who were seropositive at 7 weeks of follow-up $(n=30)$, patients with an asymptomatic COVID-19 infection $(n=12)$ and patients with a symptomatic COVID-19 infection ( $=47)$ at 7 weeks of follow-up (close contact vs asymptomatic infection, ${ }^{*} p=0.035$; close contact vs symptomatic infection $* * * p<0.00 \mathrm{I}$ ). (B) SARS-CoV-2-specific IgG antibody levels in close contacts who were seropositive at 7 weeks of follow-up $(n=30)$, patients with an asymptomatic COVID-19 infection $(n=12)$ and patients with a symptomatic COVID-19 infection $(n=47)$ at 12 weeks of follow-up (close contact vs asymptomatic infection, ${ }^{* *} p=0.004$; close contact vs symptomatic infection, $* * * 0<0.00 \mathrm{I}$ ).

confirmed COVID-19 infections. ${ }^{9}$ Among the close contacts identified in this study, only $8.3 \%$ (30/360) and $0.83 \% \quad(3 / 360)$ were SARS-CoV-2 seropositive at 7 weeks or 12 weeks of follow-up, respectively, and SARSCoV-2-specific antibody levels had decreased to baseline in $99.17 \%$ of close contacts by 12 weeks of follow-up. These findings indicate that close contacts generate a transient SARS-CoV-2-specific antibody response that rapidly wanes, providing short-lived protective immunity. In contrast, the duration of SARS-CoV-2 IgG seropositivity was stable $(\mathrm{S} / \mathrm{CO}>1)$ for 3 months in patients with confirmed COVID-19 infections. In the present study, $100 \%(12 / 12)$ and $75 \%(9 / 12)$ of patients with an asymptomatic COVID-19 infection and 95.7\% (45/47) and 93.6\% (44/47) of patients with a symptomatic COVID-19 infection were SARS-CoV-2 seropositive at 7 weeks or 12 weeks of follow-up, respectively. These data are consistent with previous reports that show high positive rates of virus-specific antibodies, an initial decrease, and sustained plateaus of low antibody levels in patients with confirmed COVID-19 infections. ${ }^{7,10}$

Entry of coronaviruses to host cells is mediated by the spike (S) glycoprotein of SARS-CoV-2. SARS-CoV-2 antibodies can recognize the $\mathrm{N}$-terminal domain and other regions on the $\mathrm{S}$ glycoprotein, which can neutralize SARS-CoV-2. ${ }^{12,13}$ Some evidence shows a positive correlation between severity of COVID-19 disease and virusspecific IgG and IgM antibody titers. ${ }^{7,11}$ In the present study, the number of SARS-CoV-2 seropositive patients with an asymptomatic or symptomatic COVID-19 infection declined over the follow-up, but antibody titers remained stable Low levels of certain antibodies have potent neutralizing capability; however, recent reports suggest that COVID-19 re-infection may occur on reexposure to SARS-CoV-2 due to a decline in antibody levels after an initial infection. 5,6,14 Extrapolating from 
A

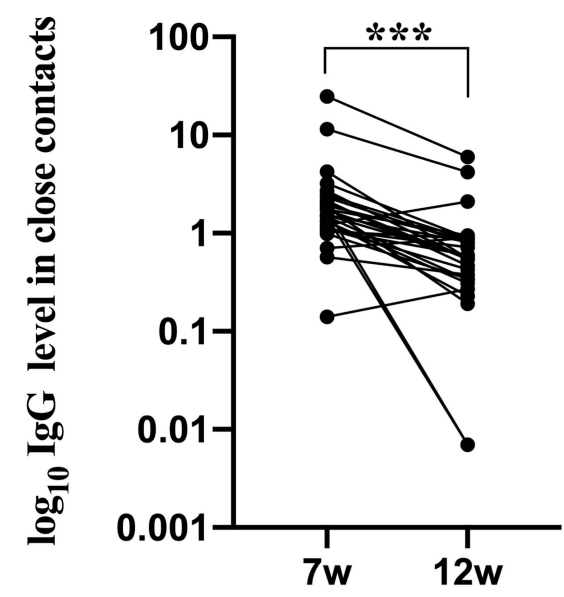

C

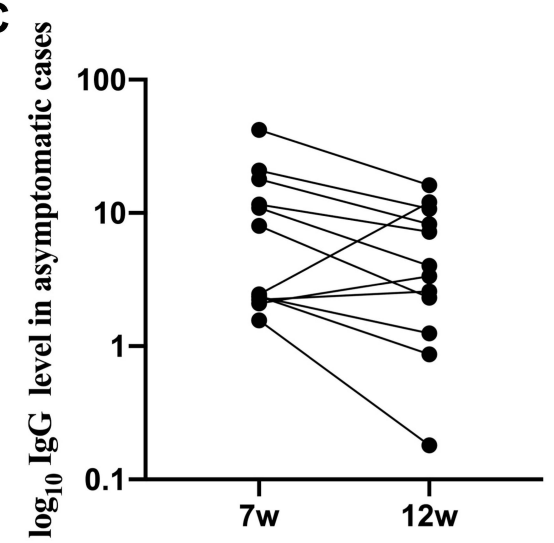

E

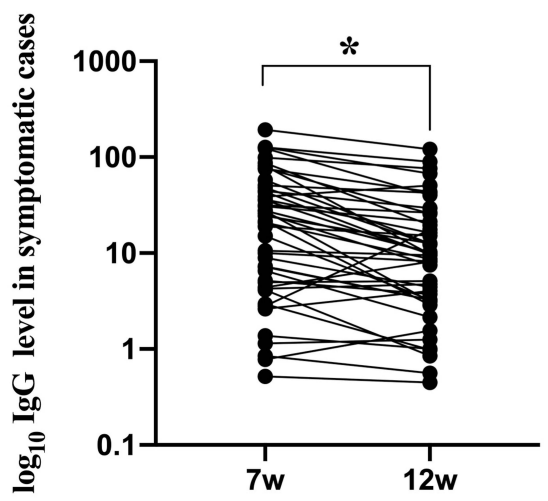

B

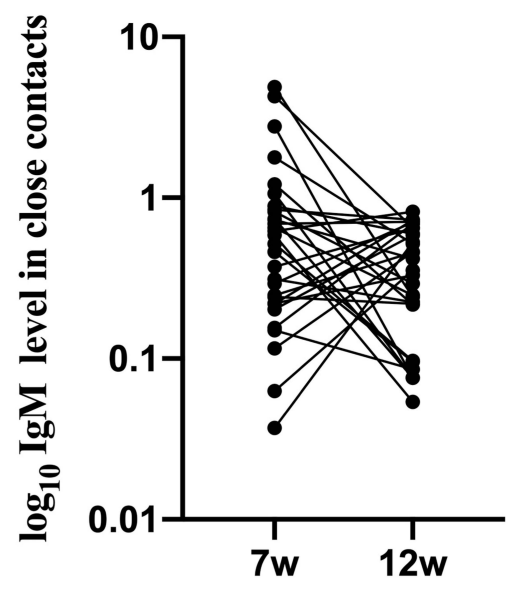

D

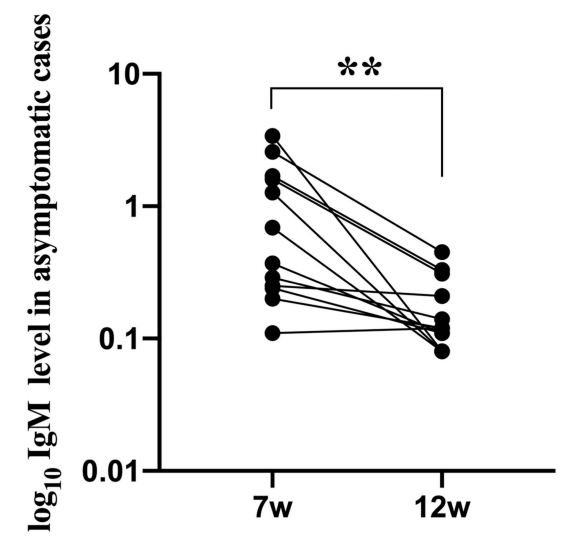

F

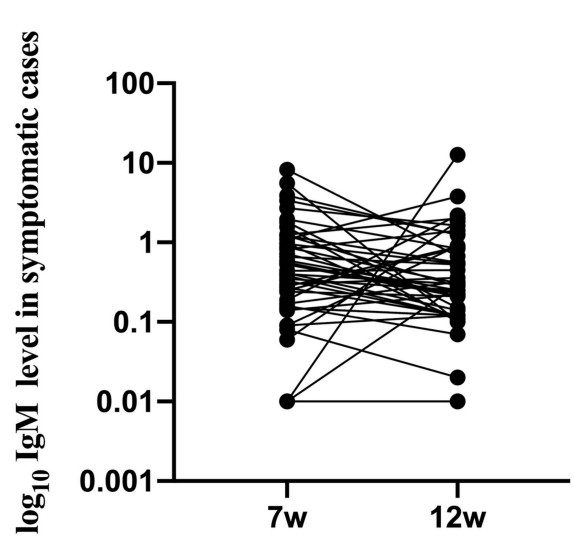

Figure 5 Change in SARS-CoV-2-specific antibody levels at 7 and 12 weeks of follow-up in close contacts who were seropositive (S/CO $\geq 1$ ) at 7 weeks of follow-up and patients with an asymptomatic or symptomatic COVID-19 infection. (A) SARS-CoV-2-specific lgG antibody levels in close contacts who were seropositive at 7 weeks of follow-up $(n=30)$ (7 weeks vs 12 weeks of follow-up in close contacts, $\left.{ }^{* * *} p<0.001\right)$; (B) SARS-CoV-2-specific lgM antibody levels in close contacts who were seropositive at 7 weeks of follow-up $(n=30)$; (C) SARS-CoV-2-specific IgG antibody levels in patients with an asymptomatic COVID-I9 infection ( $\mathrm{n}=12$ ); (D) SARS-CoV-2-specific IgM antibody levels in patients with an asymptomatic COVID-19 infection $(n=12)$ (7 weeks vs 12 weeks of follow-up in patients with an asymptomatic COVID-I9 infection, ** $p=0.003$ ); (E) SARS-CoV-2-specific IgG antibody levels in patients with a symptomatic COVID-19 infection ( $\mathrm{n}=47)(7$ weeks vs 12 weeks of follow-up in patients with a symptomatic COVID-19 infection, ${ }^{*} p=0.029$ ); (F) SARS-CoV-2-specific IgM antibody levels in patients with a symptomatic COVID-19 infection ( $\mathrm{n}=47$ ).

these reports and the findings from the current study, we suggest that low antibody titres in close contacts are unlikely to provide effective immune protection against SARS-CoV-2 over the long term.
Our findings showed that SARS-CoV-2-specific IgG and $\operatorname{IgM}$ antibody levels were significantly higher at 7 and 12 weeks of follow-up in close contacts exposed in the hospital setting compared to the social setting. 
However, at 7 weeks of follow-up, SARS-CoV-2 IgM antibody levels were significantly higher in close contacts who were exposed through a relative compared to a doctor-patient relationship, and at 12 weeks of followup, SARS-CoV-2 IgG antibody levels were significantly higher in close contacts who were exposed through a colleague compared to a doctor-patient relationship. This study was conducted in a hospital that provides healthcare to the community in Qiongzhong at the center of Hainan province. Close contacts comprised medical staff and individuals from the community. The number of close contacts of the case-patients was lower than has been reported elsewhere in China, ${ }^{2}$ possibly because the hospital was not authorized to receive patients with COVID-19, communities in Qiongzhong have low population densities, and public health measures in Qiongzhong effectively controlled local transmission. Similar to our results, SARS-CoV-2 antibody screening in 3056 health-care workers at a hospital in Belgium revealed $6.4 \%$ of study participants had SARS-CoV-2-specific IgG antibody; however, only having a household contact with suspected or confirmed COVID-19 was associated with antibody positivity. ${ }^{15}$ In New York, the prevalence of SARS-CoV -2 antibodies was significantly lower among hospital employees compared to the general public on Long Island $(9.9 \%$ vs $16.7 \%, \mathrm{P}<.001),{ }^{16}$ potentially due to safety strategies implemented in hospitals.

Importantly, and consistent with previous reports, ${ }^{17}$ we found that $56.7 \%(17 / 30)$ of close contacts aged $<18$ years were seropositive at 7 weeks of follow-up, and SARSCoV-2-specific IgG and IgM antibody levels were significantly higher in close contacts aged $<18$ years compared to close contacts aged 18-64 years. These data suggest that close contacts aged $<18$ years rapidly build immunity to SARS-CoV-2. Considering current vaccination programs in China and some other countries around the world do not include individuals aged $<18$ years old, the antibodies elicited by close contact may have a protective effect, and the spread of SARS-CoV-2 may be slower in this age group. ${ }^{18}$ An estimated $2.2 \%$ of incident COVID-19 cases occur in individuals aged $<19$ years old, clinical manifestations are usually mild, and the case fatality rate in this age group is $<0.1 \%{ }^{8}$ The infection rate among close contacts of children and adolescents is $13.2 \%$, and significantly lower than among close contacts of adults $(21.2 \%) .{ }^{19}$ This is consistent with the conclusion that high SARS-CoV-2 seroprevalence in children may have a significant protective effect. Conversely, individuals aged $<18$ years with a COVID-19 infection that do not have clinical symptoms may unknowingly expose others, increasing the risk for clusters of SARS-CoV-2 infections in schools. Public health measures, such as wearing a mask, maintaining social distance and practicing proper personal hygiene, should be implemented to prevent transmission of SARS-CoV-2 in schools. Parents, teachers and other staff in schools should be vaccinated to protect the students from infection and establish an immune barrier to limit SARS-CoV-2 transmission from school to society.

This study was associated with several limitations. First, measurement of neutralizing antibody titers was restricted due to lack of biosafety Level 3 (BSL-3) laboratory. Second, a longer follow-up is required to determine whether SARS-CoV-2-specific antibody levels continued to decline or reached a plateau. Third, the number patients with an asymptomatic COVID-19 infection was low due to the lack of an effective diagnostic test to identify these individuals in a timely manner.

In conclusion, this study showed that the SARS-CoV -2-specific antibody response conferred by close contact with an individual with a COVID-19 infection is short term and unlikely to contribute to herd immunity against SARS-CoV-2. Until the threshold of herd immunity against SARS-CoV-2 is reached, outbreaks will lead to resurgence of COVID-19 in the general population. There remains an unmet public health need for mass vaccination of populations around the world to increase levels of protective antibodies, achieve and maintain herd immunity, and curb future waves of the COVID-19 pandemic.

\section{Acknowledgments}

The authors thank the close contacts, their relatives, and health workers who willingly participated in this study. JinJian Yao, Hangfei Wang and Weiling Yu are co-first authors for this study.

\section{Funding}

This research was funded the Province Natural Science key Foundation of Hainan (No: ZDYFXGFY2020004 and No: ZDYF 2019125); the Province Natural Science Foundation of Hainan (No: 818MS131); National Natural Science Foundation of China (No 81960351).

\section{Disclosure}

The authors declare no conflict of interest. 


\section{References}

1. McMichael TM, Currie DW, Clark S, et al. Epidemiology of Covid-19 in a long-term care facility in King County, Washington. $N$ Engl J Med. 2020;382:2005-2011. doi:10.1056/NEJMoa2005412

2. WHO. WHO coronavirus disease (COVID-19) dashboard; 2021. Available from: https://covid19.who.int. Accessed April 23, 2021.

3. Meys R, Delbressine JM, Goërtz YMJ, et al. Generic and respiratory-specific quality of life in non-hospitalized patients with COVID-19. J Clin Med. 2020;9(12):3993. doi:10.3390/jcm9123993

4. Xu X, Sun J, Nie S, et al. Seroprevalence of immunoglobulin M and G antibodies against SARS-CoV-2 in China. Nat Med. 2020;26 (8):1193-1195. doi:10.1038/s41591-020-0949-6

5. Jin Y, Wang M, Zuo Z, et al. Diagnostic value and dynamic variance of serum antibody in coronavirus disease 2019. Int J Infect Dis. 2020;94:49-52. doi:10.1016/j.ijid.2020.03.065

6. Li K, Huang B, Wu M, et al. Dynamic changes in anti-SARS-CoV-2 antibodies during SARS-CoV-2 infection and recovery from COVID-19. Nat Commun. 2020;11(1):6044. doi:10.1038/s41467020-19943-y

7. Seow J, Graham C, Merrick B, et al. Longitudinal observation and decline of neutralizing antibody responses in the three months following SARS-CoV-2 infection in humans. Nat Microbiol. 2020;5 (12):1598-1607. doi:10.1038/s41564-020-00813-8

8. Long QX, Tang XJ, Shi QL, et al. Clinical and immunological assessment of asymptomatic SARS-CoV-2 infections. Nat Med. 2020;26(8):1200-1204. doi:10.1038/s41591-020-0965-6

9. Long QX, Liu BZ, Deng HJ, et al. Antibody responses to SARS-CoV-2 in patients with COVID-19. Nat Med. 2020;26 (6):845-848. doi:10.1038/s41591-020-0897-1

10. He Z, Ren L, Yang J, et al. Seroprevalence and humoral immune durability of anti-SARS-CoV-2 antibodies in Wuhan, China: a longitudinal, population-level, cross-sectional study. Lancet. 2021;397(10279):1075-1084. doi:10.1016/S0140-6736(21)00238-5
11. Lee N, Chan PKS, Ip M, et al. Anti-SARS-CoV IgG response in relation to disease severity of severe acute respiratory syndrome. J Clin Virol. 2006;35(2):179-184. doi:10.1016/j.jcv.2005.07.005

12. Lopes LR, De Mattos Cardillo G, Paiva PB. Molecular evolution and phylogenetic analysis of SARS-CoV-2 and hosts ACE2 protein suggest Malayan pangolin as intermediary host. Braz J Microbiol. 2020;51(4):1593-1599. doi:10.1007/s42770-020-00321-1

13. Liu X, Wang J, Xu X, et al. Patterns of IgG and IgM antibody response in COVID-19 patients. Emerg Microbes Infect. 2020;9 (1):1269-1274. doi:10.1080/22221751.2020.1773324

14. Edridge AWD, Kaczorowska J, Hoste ACR, et al. Seasonal coronavirus protective immunity is short-lasting. Nat Med. 2020;26 (11):1691-1693. doi:10.1038/s41591-020-1083-1

15. Steensels D, Oris E, Coninx L, et al. Hospital-Wide SARS-CoV-2 antibody screening in 3056 staff in a tertiary center in Belgium. JAMA. 2020;324(2):195-197. doi:10.1001/jama.2020.11160

16. Jeremias A, Nguyen J, Levine J, et al. Prevalence of SARS-CoV-2 infection among health care workers in a tertiary community hospital. JAMA Intern Med. 2020;180(12):1707. doi:10.1001/jamainter nmed.2020.4214

17. ChangT H, Wu JL, Chang LY. Clinical characteristics and diagnostic challenges of pediatric COVID-19: a systematic review and meta-analysis. $J$ Formos Med Assoc. 2020;119(5):982-989. doi:10.1016/j.jfma.2020.04.007

18. Zimmermann P, Curtis N. Coronavirus infections in children including COVID-19: an overview of the epidemiology, clinical features, diagnosis, treatment and prevention options in children. Pediatr Infect Dis J. 2020;39(5):355-368. doi:10.1097/INF.0000000 000002660

19. Hua CZ, Miao ZP, Zheng JS, et al. Epidemiological features and viral shedding in children with SARS-CoV-2 infection. J Med Virol. 2020;92(11):2804-2812. doi:10.1002/jmv.26180
Journal of Inflammation Research

\section{Publish your work in this journal}

The Journal of Inflammation Research is an international, peerreviewed open-access journal that welcomes laboratory and clinical findings on the molecular basis, cell biology and pharmacology of inflammation including original research, reviews, symposium reports, hypothesis formation and commentaries on: acute/chronic inflammation; mediators of inflammation; cellular processes; molecular mechanisms; pharmacology and novel anti-inflammatory drugs; clinical conditions involving inflammation. The manuscript management system is completely online and includes a very quick and fair peerreview system. Visit http://www.dovepress.com/testimonials.php to read real quotes from published authors. 